\title{
La importancia de la gestión directiva en la escuela
}

\section{The importance of directive management in primary school}

\author{
CHÁVEZ-MORALES, Ignacio †*, CHÁVEZ-LÓPEZ, Pedro Jacob, LINO-GAMIÑO, Juan Alfredo y \\ MAGDALENO-CASTRO, Benigno
}

\author{
Centro de Investigaciones Sociales y Educativas Tecomán AC (CISET). Portugal \#47 Jardines del Chamizal, Tecomán, \\ Colima. \\ Universidad de Colima, Facultad de Contabilidad y Administración de Tecomán. Avenida Universidad 333, Las Víboras, \\ 28040 Colima, Col. \\ Centro Pedagógico de Durango AC (CPD), alle Coronado Pte. 922, Zona Centro, 34000 Durango, Dgo.
}

ID $1^{\text {er }}$ Autor: Ignacio, Chávez-Morales / ORC ID: 0000-0001-9850-2989

ID $1^{\text {er }}$ Coautor: Pedro Jacob, Chávez-López / ORC ID: 0000-0003-2378-6061

ID $2^{\text {do }}$ Coautor: Juan Alfredo, Lino-Gamiño / ORC ID: 0000-0002-7022-5438, Research ID Thompson: 3059393, CVU CONACYT ID: 268945

ID $3^{\text {er }}$ Coautor: Benigno, Magdaleno-Castro / ORC ID: 0000-0002-1404-6136

DOI: $10.35429 / J B E .2019 .9 .3 .24 .34$

Recibido 12 de Julio, 2019; Aceptado 25 de Septiembre, 2019

\begin{abstract}
Resumen
El trabajo que aquí se presenta aborda la importancia del liderazgo y la gestión por parte del director en la escuela en relación a la educación que el nuevo siglo necesita; se centra esencialmente en rescatar la trascendencia del papel directivo en el liderazgo y gestión del centro educativo. Para la investigación se han planteado objetivos generales y específicos que buscan básicamente conocer y rescatar la importancia del papel que juega el director de la institución, por un lado, y por otro, conocer las diferentes teorías sobre liderazgo y gestión que conocen los directores informantes para el estudio. Para tales fines se realizó un estudio de tipo exploratorio con una hipótesis de dos variables; la importancia de la gestión y el liderazgo, para su comprobación se aplicó una encuesta (con carácter de validación) a 8 directores del Municipio de Tecomán, Colima. En la parte final del documento se detallan los resultados, hallazgos y las conclusiones, entre las cuales destaca el hecho de que es determinante el grado de actualización del director para definir su estilo de liderazgo, remarcar la importancia de su adecuada gestión de los recursos de todo tipo al interior de la escuela y para cada una de las actividades.
\end{abstract}

Gestión, Dirección, Liderazgo

\begin{abstract}
The work presented here addresses the importance of leadership and management by the principal in the school in relation to the education that the new century needs; it is essentially focused on rescuing the significance of the leadership role in the leadership and management of the school. For the research have been set general and specific objectives that seek basically to know and rescue the importance of the role played by the director of the institution, on the one hand, and on the other, to know the different theories about leadership and management that informing directors know for the study. For these purposes, an exploratory type study was carried out with a two-variable hypothesis; the importance of management and leadership, for verification, a survey (as validation) was applied to 8 directors of the Municipality of Tecomán, Colima. The final part of the document details the results, findings and conclusions, among which the fact that the degree of updating of the director is decisive to define his leadership style, highlighting the importance of his proper management of resources of all kinds within the school and for each of the activities.
\end{abstract}

Management, Direction, Leadership

Citación: CHÁVEZ-MORALES, Ignacio, CHÁVEZ-LÓPEZ, Pedro Jacob, LINO-GAMIÑO, Juan Alfredo y MAGDALENO-CASTRO, Benigno. La importancia de la gestión directiva en la escuela. Revista de Educación Básica. 2019. 3-9: 24-34.

\footnotetext{
*Correspondencia al Autor (Correo Electrónico: sflores@utaltamira.edu.mx)

$\dagger$ Investigador contribuyendo como primer autor.
} 


\section{Introducción}

El director de escuela es un agente importante porque se encarga de implementar las Políticas Educativas como Públicas en la escuela. Para esto la empatía que pueda tener con los maestros, padres de familia, alumnos como con las diferentes representaciones de las instituciones permite generar la confianza entre los diferentes agentes que frecuentan la institución. La Educación que exige el siglo XXI tiene que ver con el funcionamiento, liderazgo y gestión de quien dirige las instituciones en este sentido, hay poca formación en los directores y menos se considera su importancia, tal como lo deja ver Pont al afirmar que "El liderazgo en los centros está abandonado y la formación de los directores de escuela es prácticamente nula" (Beatriz Pont, 2019, p. 27).

El funcionamiento de la Institución educativa sea del nivel que sea, depende de la administración, organización, el liderazgo la gestión que realiza el director.

\section{Objetivos}

El objetivo General - conocer y rescatar la importancia del papel que juega el director de la institución a través del liderazgo y la gestión al realizar su función.

Objetivo específico -Conocer la importancia que le dan al liderazgo y la gestión los directores de escuela del Municipio de Tecomán en la función directiva.

Conocer las diferentes teorías sobre liderazgo y gestión que conocen los directores de escuela del municipio de Tecomán.

\section{Desarrollo}

El liderazgo es una forma de conducir, dirigir, apoyar.

Gestionar - hacer diligencias para lograr un negocio a un fin. (Oceano,1991, p. 520)

a) Liderazgo a).- Daniel Goleman, "el líder debe conocerse a sí mismo y tener una autoconciencia" (Goleman, 2014, p. 35.) dentro de ello saber controlar sus emociones, una persona autoconsciente al controlar sus emociones puede hacer una crítica reflexiva y tener claro sus objetivos y sus valores. Goleman señala que la persona autoconsciente sabe hablar de manera precisa y correcta, son autorregulables lo que permite que sea además organizado en todo su proceder. (Goleman, 2014.)

En el liderazgo es fundamental la empatía, señala el autor mínimo en tres puntos que son importantes:

- El incremento en la utilización de los equipos.

- El rápido avance de la globalización.

- La necesidad de conservar los buenos trabajadores.

El líder debe ser capaz de notar y comprender los puntos de vista de sus subalternos, conducir con acierto y esto se logra con la empatía. Quien tiene una capacidad social tiene un círculo muy grande de conocidos y facilidad para encontrar puntos en común. Por lo anterior se puede decir que los líderes marcan estrategias, motivan, crean una misión e implantan una cultura (Goleman, 2014). El autor considera estilos de liderazgo:

- Autoritarios - que movilizan a la gente con una visión.

- Conciliadores - generan vínculos emocionales y armonía.

- Democráticos - crean consenso mediante la participación.

- Ejemplarizantes - esperan rendimiento y autonomía.

- Los Coach - contribuyen al desarrollo de la gente para el futuro.

- Los coercitivos - exigen una sumisión de inmediato.

Se puede observar por la definición y entender que, según sea el estilo es el tipo de liderazgo que ejerce un líder en su espacio. 
Sergiovanni señala las fuerzas del liderazgo son importantes para que una escuela tenga buenos aprendizajes, entre estos se pueden mencionar los siguientes:

- La fuerza del liderazgo técnico y humanista.

- Los liderazgos educativo, simbólico y cultural.

- Cuanto mayor sea la presencia del liderazgo es mayor la jerarquía" (Murillo et al, 2000, p. 100).

Sergiovanni numera que el director de la escuela es uno de los elementos clave para que una escuela funcione bien y tenga buenos aprendizajes, define algunos aspectos del liderazgo diciendo que "son fuerzas que pueden utilizar tanto los directores e inspectores como los profesores, que influyen en la vida de los centros y que constituyen una energía capaz de llevar una situación al cambio" (1999, p.99); plantea cinco tipos de liderazgo:

El líder técnico, hace hincapié en conceptos, técnicas de planificación y de distribución del tiempo, el directivo es el encargado de planificar, organizar, coordinar y establecer el horario de las actividades escolares.

El líder humano, concede mayor importancia a las relaciones humanas, a la competencia y en suma, a las técnicas de motivación, instrumentar y especialmente a apoyar, animar y propiciar oportunidades de desarrollo profesional a los miembros de la organización.

El líder educativo, utiliza conocimientos y opciones de profesional en la medida en que éstos se relacionan con la eficacia docente, el desarrollo de programas de supervisión, de diagnóstico, de formas de evaluación o bien de desarrollo del currículo.

El líder simbólico, asume el papel de jefe y pone énfasis de la selección de metas y comportamientos señalando día con día lo más importante, se encuentra en contacto directo con los alumnos, le interesa la cesión de la escuela, decirse de momias, rituales y otras ocasiones importantes proporciona una visión unificada del centro.
El líder cultural, que éste se caracteriza por decidir, fortalecer y articular aquellos valores, creencias y raíces culturales y ramales, su identidad. Se encarga de crear un estilo organizativo, lo que define a la escuela como una entidad diferenciada, crea una cultura propia con la actividad social.

Tudesco (1997) al hablar sobre el trabajo colectivo con el liderazgo compartido, aborda que las reformas actuales de la gestión educativa tendientes a estimular la autonomía de las escuelas a través de proyectos intentan, desde este punto introducir el trabajo en equipo y la acumulación de experiencias. Además, señala que: "un cambio como este tiene consecuencias en el trabajo de los docentes; como reconocer que en la escuela no existe un solo equipo, sino que hay más de un equipo en ellas" (Tudesco,1997, p.23) de donde saldrá un equipo dominante y la inclinación hacia el trabajo.

En ese mismo punto Fullan y Hargreaves citan a Rosenholtz "la mayoría de los docentes y directores se aíslan en su lugar de trabajo tanto que ni se tratan profesionalmente" (Fullan et al, 2000, p. 30). En cuanto al trabajo en equipo, se asocia a normas y oportunidades que dan sustento a la mejora sostenida y al aprendizaje permanente, sostienen que la mejora de la enseñanza constituye una acción más colectiva que individual, donde el liderazgo y la gestión en la escuela por parte del director son fundamentales.

De aquí surge la idea de la importancia del trabajo directivo, lo que aborda el líder como elemento transformador de la práctica del liderazgo y la gestión educativa. Javier Murillo, Raquel Barrio, José Pérez - Albo, hablan sobre el liderazgo y señalan que las investigaciones relacionadas con las direcciones, es el que se refiere sobre todo al liderazgo. 
Siguiendo a Coronel (1996) es posible esbozar la evolución de este movimiento en un primer momento, en la década de los treinta y cuarenta pues, desarrolló la teoría de los rasgos (Murillo, 2000), la cual buscaba la personalidad ideal del líder partiendo de la premisa de que los líderes nacen, no se hacen, sin embargo los estudios realizados no pudieron demostrar tal hipótesis y de hecho se encontró, que no existen características de personalidad física o intelectuales relacionados con medidas, ni siquiera existe el líder ideal, sino que siempre está ligado al contexto.

A partir de este fracaso de la definición de las características de líder se desarrolló la teoría conductual. Que define el liderazgo en términos de conducta. Los trabajos realizados al amparo de esta corriente han ofrecido una gran cantidad de conocimientos útiles, para la comprensión del fenómeno del liderazgo.

Actualmente existe una nueva corriente sobre el liderazgo, teoría de la contingencia o situación, que aspira a percibir un estilo de liderazgo adecuado, como las relaciones del líder, los miembros seguidores de sí mismo, el clima o cultura, organización y otros factores ambientales. De esta teoría se concibe al directivo escolar como agente transformacional de la cultura organizativa del centro (Bass, 1985) o como elemento facilitador que allana el camino y encauza la acción de los demás.

Es por esto que el director es una pieza fundamental para el caminar de la escuela desde dos ámbitos fundamentales: administrativo y pedagógico.

- En lo administrativo porque no se puede dejar la administración de la escuela, pues esta se mueve con un control estadístico y de resultados a través de documentos que permiten dar seguimiento al proceso enseñaza-aprendizaje de los alumnos.

- En lo pedagógico porque son los responsables de este proceso lo cual lleva a revisar métodos, técnicas, materiales de los docentes para revisar el proceso de la enseñanza.
Luego entonces, la responsabilidad de asumir el liderazgo es constante, sin interesar qué tipo de liderazgo se ejerce, pero sí, comprender que su labor es muy importante. Con esto, se dice "que dentro de los planteles hay compañeros que asumen, liderazgos, por la amistad que guardan con todos sus compañeros" (Chávez, 2005, p.33) lo cual lleva a señalar que se deben de cambiar los estilos de liderazgo o ampliar el concepto a una generalidad y no solo a una persona. Por otro lado, decir que el liderazgo es importante para lograr la gestión y que ambos conceptos no son aislados, sino que ambos se complementan. A este respecto Pastrana Flores (1994) dice que en este proceso conceptual la construcción de la definición de gestión demandó enfrentamientos con las teorías administrativas, organizativas, sociológicas y política principalmente, las que junto al énfasis pedagógico coadyuvaron a definir un punto de equilibrio. Es para Coronel (1996) que ante la necesidad de dar mayor poder al concepto y reconocer la necesidad de ampliar y llevar hasta los profesores y a otros miembros del centro y a la vez ser agentes del cambio cuando aparecen enfoques del liderazgo pedagógico como:

Liderazgo instruccional. Es parte del pedagógico cuando se trata de hablar de situaciones especiales de alguna materia, o de supervisión eficacia, evaluación de los profesores, control de la calidad y su actuación es limitada según el campo. Los autores Coronel y Bass mencionan que Hallinger (1984) mide el liderazgo pedagógico de los directores de escuelas primarias y secundarias bajo tres dimensiones como las siguientes:

\begin{tabular}{|c|c|}
\hline Dimensiones & Funciones \\
\hline $\begin{array}{l}\text { Definición del proyecto } \\
\text { del centro }\end{array}$ & $\begin{array}{l}\text { Determinar los objetivos } \\
\text { del centro y comunicar } \\
\text { dichos objetivos }\end{array}$ \\
\hline $\begin{array}{l}\text { Dirección del programa } \\
\text { pedagógico }\end{array}$ & $\begin{array}{l}\text { Supervisar y evaluar la } \\
\text { instrucción, coordinar el } \\
\text { proyecto curricular, } \\
\text { seguir la evolución de los } \\
\text { alumnos. }\end{array}$ \\
\hline $\begin{array}{l}\text { Promoción de un clima } \\
\text { escolar de aprendizaje } \\
\text { positivo }\end{array}$ & \begin{tabular}{lrr} 
Proteger & el & tiempo \\
lectivo, & promover & el \\
desarrollo & \multicolumn{2}{c}{ profesional, } \\
mantener & una visibilidad \\
alta, & proporcionar \\
incentivos & a & los \\
profesores, & proporcionar \\
incentivos & por & el \\
aprendizaje. & &
\end{tabular} \\
\hline
\end{tabular}

Tabla 1 Dimensiones del liderazgo

Fuente elaboración de los autores ya mencionados, 1984 
Cuando emergió el concepto de liderazgo instruccional, los directores y directoras se consideraban eficaces si promovían cambios en la escuela generando expectativas claras, manteniendo una disciplina firme y consiguiendo resultados elevados.

Lashway (1995) señala que a partir de los noventa hubo la necesidad de optar otras concepciones de liderazgo más sofisticadas y de acuerdo a las nuevas corrientes empresariales de esta manera aparece, el liderazgo transaccional y transformacional, que enfatiza la colaboración y la distribución del poder, el término liderazgo transformacional, implica una cualidad personal, una habilidad para inspirar a los miembros de la organización para mirar más allá, de sus propios intereses y centrarse en metas.

- El modelo transformacional pone su atención en, aumentar la capacidad de la organización de innovar a través de un énfasis en la distribución del poder, el liderazgo compartido y el aprendizaje organizativo, este modelo sugiere que los efectos de la acción del directivo se logran fomentando metas del grupo. En este mismo sentido del liderazgo transformacional Bass (1985) lo define bajo las siguientes dimensiones:

Carisma, que consiste en el poder referencial y de influencia, un líder carismático es capaz de entusiasmar e inspirar confianza e identificación con la organización. Señalan Leithwood y Steinbach (1993) la aplicación de este tipo de liderazgo en la escuela se base en tres constructos:

- La habilidad del director para fomentar el trabajo colegiado.

- El desarrollo de metas explicitas, compartidas, moderadamente desafiantes y factibles.

- Y la creación de una zona de desarrollo próximo para el directivo y su personal.
Por lo tanto, la evidencia de este liderazgo se encuentra en los medios que los directores usan para generar mejores soluciones a los problemas de la escuela, para desarrollar en los profesores compromisos con la puesta en marcha de esas soluciones y para fomentar el desarrollo del personal, una síntesis se presenta la siguiente tabla:

\begin{tabular}{|l|lll|}
\hline Dimensiones & \multicolumn{3}{|c|}{ Actuaciones } \\
\hline Propósitos & $\begin{array}{l}\text { Desarrolla una visión que es } \\
\text { ampliamente compartida por la } \\
\text { escuela. } \\
\text { Establece el consenso en los } \\
\text { objetivos y las prioridades de la } \\
\text { escuela. }\end{array}$ \\
\hline Personas & $\begin{array}{l}\text { Presta apoyo individual } \\
\text { Presta apoyo Intelectual } \\
\text { Ofrece modelos de ejercicio } \\
\text { profesional. }\end{array}$ \\
\hline Estructura & $\begin{array}{l}\text { Distribuye la responsabilidad y } \\
\text { comparte la autoridad del liderazgo. } \\
\text { Posibilita tiempo para la } \\
\text { planificación colegiada. }\end{array}$ \\
\hline Cultura & $\begin{array}{l}\text { Fortalece la cultura en la escuela } \\
\text { Favorece el trabajo en la } \\
\text { colaboración lan } \\
\text { Entabla comunicación directa y } \\
\text { frecuente y } \\
\text { Utiliza símbolos para expresar los } \\
\text { valores culturales. }\end{array}$ \\
\hline
\end{tabular}

Tabla 2 Síntesis del ejercicio del liderazgo transformacional

Fuente: elaborado a partir de lo que señala Leithwood. 1993

La concepción del liderazgo transformacional ha ido evolucionando y dando paso a un nuevo modelo, el liderazgo facilitador, "donde la tendencia es ejercer el poder a través de los demás, no sobre ellos, de manera que puede ser definido como los comportamientos que favorecen la capacidad colectiva de una escuela de adoptarse, resolver problemas y mejorar sus resultados" (Conley y Coleman, 1994, p.58) al punto de hacer de las estrategias para prever limitaciones de recursos, construir equipos, coordinar y gestionar.

Pam Sammons, Josh Hillman, Peter Mortimore (1994) cuando hablan sobre liderazgo dicen que es un factor clave, argumentan señalando a Gray que "la importancia del liderazgo de la dirección es uno de los mensajes más claros que nos ofrece la investigación sobre la efectividad escolar" (Hallingeer y Lethwood, 1994, p. 103). 
Señalan que el hecho de que no haya surgido evidencia alguna de escuelas efectivas con liderazgo débil, en las revisiones de las investigaciones sobre efectividad, la importancia del liderazgo del director $\mathrm{o}$ directora, más que de los otros miembros del cuerpo académico son muy importantes, ya que existe marcada influencia en los siguientes elementos: Un liderazgo profesional que se caracteriza por ser firme y dirigido con un enfoque participativo; y ser guiado por un profesionista sobresaliente. Hallingeer y Leithwood (1994) marcan que el liderazgo no se relaciona con la calidad de los líderes individuales, aunque esto sea importante, puntean que, resulta fundamental el papel que juegan los líderes, su estilo, su relación con la visión, valores y metas de la escuela y su manera de abordar el cambio, los directores deben encontrar el estilo y las estructuras más adecuadas a su propia situación local, algunas características que señalan:

- Fuerza en los propósitos.

- Involucrar al cuerpo académico en la toma de decisiones.

- Autoridad profesional en los procesos de enseñanza y de aprendizaje.

Sammons, Hillman, Mortimore (1994) cuando hablan de los directores y al referirse a que debe ser:

- Firme y dirigido, el liderazgo, con propósito, los líderes excepcionales suelen ser dinámicos y logran formar un gran equipo de trabajo conjuntando las fuerzas de los docentes para iniciar el cambio. Además, un aspecto fundamental del liderazgo firme es la intercesión, la habilidad de mediar o amortiguar los agentes de cambio negativos, de desafiar y hasta violar lineamientos externos. (Levine y Lzotte, 1990; Hopkins, Ainscow y West,1994). Por esto se dice que el papel clave del liderazgo para iniciar y mantener el proceso de mejoramiento de las escuelas es importante.

- Sobre el enfoque participativo, es una característica de los directores líderes es compartir las responsabilidades del liderazgo con otros miembros del grupo o equipo y de involucrar de manera más general a los maestros en la toma de decisiones.
Los autores señalados mencionan que estas dos primeras características del liderazgo requiere claridad, evitar toda autocracia, así como la excesiva democracia para trabajar, juzgar cuidadosamente cuando tomar una decisión y cuando involucrar a otros.

- Profesionista sobresaliente en la mayoría de los casos el director no es el simple administrador, sino que en cierto sentido es un profesionista sobresaliente, que se involucra y conoce lo que sucede en el aula, incluyendo el currículo, estrategias de enseñanza y seguimiento del proceso de los alumnos.

- Además implica que el director debe proyectar un perfil de altura por medio de acciones; como desplazarse frecuentemente por la escuela, visitas las aulas y conversaciones informales con los maestros (Sizemore 1983, Montimore 1988, Pollack 1987, Teddlie 1989). El director debe también evaluar la forma en que funcionan los maestros, sobre estos elementos los autores mencionan a Scheerens "todo esto es uno de los pilares del liderazgo educacional" (Pam Sammons, 1998, p.30), se dice que la influencia de los niveles de desempeño y avance de los estudiantes operan en forma directa, al influir en la cultura de la escuela y del personal, actitudes y comportamiento que, a su vez, afectan las prácticas en el aula y la calidad de la enseñanza y el aprendizaje.

Silvia Schmelkes (2000) cuando aborda el tema sobre liderazgo, habla sobre la calidad de la educación, manifiesta que el director debe ser líder pues, debe apoyar y estimular, siendo este, su papel principal, el director debe fungir como un auténtico líder, satisfacer cada vez mejor a los beneficiarios de la escuela, hacer sentir al docente orgulloso de su trabajo. Señala "que el líder en vez de ser juez que inspecciona y evalúa a las personas, es un compañero que aconseja y dirige a su gente día a día, aprendiendo de ellos y con ellos, pues el objeto del liderazgo es mejorar el comportamiento del ser humano para mejorar la calidad de la educación" (Schmelkes, 2000.p. 123).

b) La gestión 
La gestión elemento fundamental en el liderazgo de las escuelas." El colectivo creemos que la gestión se mueve en ámbitos donde el primer responsable es el director de la escuela, o así tradicionalmente se ha concebido, pero observamos que el director como gestor se mueve entre las diferentes situaciones a las que llamamos dimensiones, ser director de escuela no es lo mismo dirigir que una empresa, ambas cosas son diferentes." (Chávez, 2005, p.109).

Las dimensiones en que observamos nos movemos; es en lo social pues interaccionamos con la comunidad escolar, así como con los compañeros docentes, y los padres de familia participando como agentes de la sociedad en mutable transformación; en lo sindical que como trabajador de la educación nos favorece ese derecho participando políticamente como parte de una organización para defender los derechos de cada uno de nosotros (Chávez,2005) por ello se plantean las siguientes dimensiones.

En lo económico porque nuestra situación y ubicación de nuestro trabajo no es ajena a la situación del entorno familiar la cual repercute indirectamente en los alumnos de nuestras escuelas.

En lo cultural porque la escuela es la generadora de conocimientos y la propulsora de la cultura hacia la comunidad y los responsables de los centros de trabajo.

De lo pedagógico porque como directores nos movemos en este ámbito en nuestras escuelas aportando y dando ideas en la cuestión teórica y metodológica a los compañeros.

Por último en la dimensión administrativa que por nombramiento o por la comisión se nos asignó, somos los inmediatos responsables del funcionamiento de las escuelas, papel que desempeñamos en unión de todos los demás, por esto afirmamos que los directores nos movemos en la gestión en diferentes dimensiones y que nuestras funciones son multidimensionales lo cual nos hace estar en contacto con todo nuestro entorno (Chávez, 2005).
Pastrana expresa que los estudios de la gestión escolar han cobrado fuerza durante los años ochenta y los noventa en diversas latitudes, incluidos de los países latinoamericanos, recientemente estos estudios han sido llamados como "temas emergentes" (Pastrana,1994, p. 128). Para aclarar el concepto menciona dos perspectivas teórico prácticas relevantes para pensamiento socioeducativo contemporáneo.

- Una proveniente de la teoría gerencial que la definen como Badajoz, se va a dirigir las instituciones educativas conforme a un modelo administrativo empleo por la empresa (Ball, 1993.)

- Otra se derivada del movimiento social analítico de la pedagogía institucional francesa que postula la autogestión o autogobierno de las instituciones. (Furlan, 1994)

La teoría gerencial supone que el devenir escolar está prescrito por decisión del director o conductor del plantel, quien a su vez transmite y cuida del cumplimiento de órdenes, que provienen de arriba. Por otro lado, la teoría social - analítica supone la participación activa y deliberada de los miembros para decidir el rumbo institucional.

Lo anterior permite pensar a la gestión como una totalidad compleja, que cobra existencia histórica como plano de articulación de los heterogéneos componentes que soportan la operación diaria de las escuelas. La gestión involucra simultánea y proporcionalmente a las dimensiones políticas, administrativas y organización en el plano cotidiano.

Por esto se puede señalar que la gestión escolar, cobra vida mediante procesos de articulación armónica de las dimensiones política, administrativa, de organización y pedagógica. En suma, gestión es un concepto de composición plural y diversa que permite apreciar la síntesis multidimensional, en este caso corresponde a la dirección jugar el papel de eje articulador de los componentes que constituyen a la gestión, por lo que la figura del director es central para conducir el plantel, Ezpeleta (1991) la expresa del siguiente modo: 
Toca a la dirección de la escuela, "núcleo de la gestión del establecimiento, articular las estrategias que, en definitiva, son las que perfilan las prioridades para la acción del cuerpo docente de cada escuela" (Ezpeleta 1991, p. 48). Definiendo de esta forma concreta se encuentra un punto de articulación en la dirección a la conducción escolar, como una construcción local, no autodefinida y relativamente consensada, que constituye la dinámica del establecimiento para que allí tenga cabida la docencia. Gimeno Sacristán (Ezpeleta, 1991) señala que la gestión de los procesos escolares es común del gobierno, pensando en los elementos que configuran la dinámica de los fenómenos que ocurren en el seno de los centros escolares, por otro lado, ha planteado que la gestión pedagógica es un concepto pertinente a la escala de las unidades escolares, propuestos por el sistema y las concreciones de la actividad escolar.

Pastrana (1994) señala que desde la perspectiva de la Policy - making, puede pensarse a la gestión escolar como expresión concreta, contextualmente definida tanto por la organización local del trabajo como el entorno que adoptan los postulados orientadores de la política estatal en materia educativa, por lo tanto, podemos considerar la gestión como algo local. Es por ello que abordar el tema de la gestión implica tratar los problemas inherentes a cualquier acción colectiva de los seres humanos donde las perspectivas parecen bifurcarse planteando:

- "La determinación del peso de las estructuras sobre otras dinámicas.

- La convicción de que los sujetos definen cabalmente sus condiciones de encuentro con los otros individuos" (Pastrana,1994, p.178).

Es por esto, que es importante definir que optar como punto de equilibrio para no caer a polarizar una gestión, sino, que los individuos definan a su libre albedrío y esto, porque la organización escolar ha sido desconocida e ignorada por las concepciones más difundidas con respecto a la gestión. Con esto señala Pastrana, que los análisis educativos todavía eluden la importancia que la dinámica interna escolar tiene para el desempeño docente y la conducción directiva de cada una de las escuelas.
Namo de Mello afirma "que la gestión es el conjunto de estrategias diferenciadas, dirigidas a la solución de problemas que deben claramente identificadas y caracterizadas" (Namo de Mello,1998, p.58). A diferencia de la planificación que tiende a realizar previsiones a más largo plazo, el modelo de gestión aspira a objetivos de corto y mediano plazo.

La gestión escolar para el departamento de investigación de la Secretaría de Educación Pública es "una de las instancias de toma de decisiones acerca de las políticas de educativas del país" (S.E.P. 2000, p.8) la gestión escolar realiza las políticas educacionales en cada unidad educativa adecuando al contexto y a las particularidades y necesidades de su comunidad educativa.

$\begin{array}{ll}\text { Gestión } & \begin{array}{l}\text { Aprendizaje } \\ \text { de niños y } \\ \text { escolar }\end{array} \\ \text { jóvenes }\end{array}$

\section{Figura 1}

Fuente: S.E.P Antología de la Gestión Educativa 2000

La idea fundamental de la gestión escolar es dinamizar los procesos y la participación de los actores que intervienen en la acción educativa por esto que recupera:

- La globalidad de la institución.

- La intencionalidad pedagógica y educativa.

- Incorpora a los sujetos de la acción educativa como protagonista del cambio educativo.

- Se da a la tarea de construir procesos de calidad para lograr los resultados buscados. (S.E.P. 2000).

Se plantean algunas dimensiones como:

- La dimensión pedagógico - curricular, hace referencia a los fines, a los objetivos específicos o razón de ser de la institución escuela en la sociedad.

- La dimensión comunitaria, apunta a la relación entre sociedad y escuela y específicamente entre la comunidad local y su escuela, relación con los padres de familia, así como la participación de las fuerzas vivas. 
- La dimensión administrativa- financiera, incorpora el tema de los recursos necesarios disponibles o no, con vistas a lo obtención, distribución, articulación y optimización para la consecución de la gestión de la institución educativa.

- La dimensión organizacional - operativa, constituye el soporte de las anteriores dimensiones proponiéndose articular su funcionamiento.

\begin{tabular}{lc} 
Gestión escolar & $\begin{array}{c}\text { Pedagógico } \\
\text { Curricular }\end{array}$ \\
Equipo Directivo & Unizizajes de \\
niños jóvenes & Comunitario \\
& $\begin{array}{c}\text { Administrativo } \\
\text { Financiero }\end{array}$ \\
\hline Figura 2 & $\begin{array}{c}\text { Organizacional } \\
\text { Operativo }\end{array}$
\end{tabular}

Figura 2

Fuente: S.E.P Antología de la Gestión Educativa 2000

El departamento de investigación de la S.E.P. intenta una gestión escolar bajo los principios de ser:

- Integral.

- Consciente.

- Transformadora.

- Participativa.

Ya que señala que "la gestión escolar no es una construcción arbitraria y aislada, pues necesariamente reconoce su pertenencia a un ámbito social especifico" (S.E.P, 2000, p.15). La gestión escolar no se asienta sólo un su propio espacio pedagógico y logístico, sino que parte de un dominio social que le da sentido y contundencia como proyecto de transformación de los seres humanos.

\section{Hipótesis Única}

El liderazgo y la gestión del director de una institución educativa es fundamental ya que ésta, permite que quienes participan sean motivados para las actividades que se desarrollan en la institución.

\section{Metodología}

\section{Tipo de trabajo exploratorio}

Los estudios exploratorios sirven para aumentar el grado de familiaridad con fenómenos, obtener información sobre la posibilidad de llevar a cabo una investigación, sobre un contexto particular de la vida real, investigar problemas de comportamiento humano, documentar experiencias, examinar temas, tendencias, identificar relaciones potenciales entre variables para investigaciones posteriores.

- Universo. Directores de educación primaria.

- Muestra- Directores de educación primaria del Municipio de Tecomán Col.

- Instrumento - Encuesta Mixta con preguntas cerradas y dos preguntas abiertas.

Descripción de la encuesta - la encuesta se compone de seis preguntas cerradas con un esquema de opción múltiple y dos opciones abiertas (ver anexo). Cabe señalar que la encuesta aplicada cumple la etapa de validación del instrumento, el cual se podrá modificar cuando el estudio se amplié, como está contemplado, pero por el momento sirve solo para tener un acercamiento a la comprobación de la hipótesis.

\section{Resultados}

Respuesta a las afirmaciones

1.- Los encuestados 8, por lo que aparece en los resultados todos asumen el liderazgo en la escuela.

2.-Sobre el tipo de liderazgo 6 directores asumen como democráticos y dos que son conciliadores.

3.-Sobre las teorías del liderazgo, conocen el liderazgo democrático 7 y uno la teoría técnica - humanista.

4.- Sobre cómo se entiende el liderazgo compartido los 8 lo comprenden.

5.-La gestión como parte del liderazgo los 8 asumen que se puede hacer gestión sin ser líder. 
6.-La forma en que se entiende la gestión, 4 sostiene que es parte del liderazgo, 3 es una forma de buscar apoyos, 1 administrar la escuela.

Al resumir la afirmación 7 se concluye:

Líder es quien dirige, comparte y devenga, liderazgo y gestión se complementan, el líder es honesto y capaz. La razón por la que es importante el director; La importancia dirigir y compartir, asumir liderazgo, coordina, es importante para la organización, orienta acciones.

\section{Discusión}

Retomando a Goleman acerca de estar consciente sobre el papel que juega el liderazgo, por los resultados se observa que los directores son consientes de su función y asumen el liderazgo, se observa que conocen algunas teorías sobre los tipos de liderazgo como de (Goleman,2014) (Sergiovanni,1999) pues hacen mención sobre el enfoque técnico y humanista reconociendo teorías, a la vez reconocen el liderazgo compartido que se ejerce como la gestión (Chávez,2005). Se reconoce que el liderazgo y la gestión van unidas y forman un elemento fundamental tal y como lo señala Pastrana.

\section{Conclusiones}

Según los resultados y las teorías revisadas los directores asumen su liderazgo, cada uno según su entorno laboral y la formación y experiencia con que cuentan para ejercerlo. Los directores en las escuelas ordenan las acciones, coordinan los esfuerzos y gestionan para la mejora en las escuelas, lo cual muestra la importancia del director, esto permite hacer un acercamiento a la hipótesis planteada como a los objetivos que se plantearon.

Uno de los puntos más importantes es que, considerando toda la información teórica y las aportaciones que realizaron los directivos escolares, es que es imprescindible tener el conocimiento teórico que se va actualizando día a día.
Con lo anterior se quiere hacer notar que aquellos directivos que desconocen los nuevos hallazgos y propuestas para mejorar el liderazgo escolar, cuentan con recursos limitados para ejercer su función, la visión se sesga y la realidad puede estarse interpretando de manera inadecuada puesto que las sociedades y sus dinámicas sociales van cambiando continuamente y con ello se ve afectado también la dinámica de los centros educativos. Cuantos más enfoques y perspectivas se conozcan, más posibilidades de efectividad se tendrán para destacar la importancia de la labor del directivo en su escuela.

Por otro lado, a partir de lo que aquí se ha manifestado se pretende realizar un estudio más extenso que pueda aportar más datos con los que se logre profundizar en el análisis y la comprensión del tema de la investigación. Por ejemplo, realizar un estudio en todo un municipio y en el mejor de los casos a nivel estado. Junto a lo anterior, el presente tema permite también la posibilidad de indagar en otros temas relacionados a él y que son de suma importancia en la actualidad del estado, por ejemplo: conocer en qué medida los directivos están actualizados en cuanto a las teorías del liderazgo, cuáles son los factores que coadyuvan y cuáles son los que impiden que la acción directiva se lleve a cabo de manera efectiva y se traduzcan en la mejora de los aprendizajes de los estudiantes, qué grado de importancia tiene la actualización del directivo y no solo del docente para las autoridades educativas; entre otros, todos temas de notable urgencia.

\section{Referencias}

Antúnez Marcos, S. (2002). La autonomía en las escuelas. Muralla. España.

Chávez Morales, I. (2005). Liderazgo más participación igual a autonomía. Voluntades. Morelia, Mich.

Coronel, J. (1999). La dirección escolar. M.E. CIDE. Madrid.

Fullan, M. y Hargreaves, A. (2000). La escuela que queremos. SEP, México.

Rafael Furlan, Rodríguez. (1994) Citan A. Delorme. La pedagogía institucional. Esfinge. Barcelona.

CASTILLO-FLORES, Esther Sarai \& DE LA CUESTA-SOLIS, Edgar. Educación financiera como modelo de inclusión financiera para empoderamiento de grupos vulnerables. Revista de Educación Básica. 2019 
Goleman, D. (2014). Liderazgo el poder de la inteligencia emocional. Ed. B

Murillo Torrecilla, F., Barrio Hernandez, R., Pérez Albo, M. La dirección escolar. Ministerio de educación y cultura. CIDE. Madrid España. 1999.

Namo de Mello, G. (1998). Nuevas propuestas para la gestión educativa. S.E.P México.

Pastrana, L. (1994). La gestión escolar. Cide. México.

Schmelkes, S. (2000). Hacia la calidad de la educación en las escuelas. S.E.P. México.

S.E.P. Antología de gestión educativa. SE.P. México. 2000.

Tudesco, J. (1997) Comentarios a la $45^{\circ}$ Conferencia Internacional de la Educación. Cuadernos biblioteca para la actualización del maestro. SEP. México.

Pont, B. (2019). El país Madrid. analista de políticas educativas de la OCDE 